Esta revista forma parte del acervo de la Biblioteca Jurídica Virtual del Instituto de Investigaciones Jurídicas de la UNAM

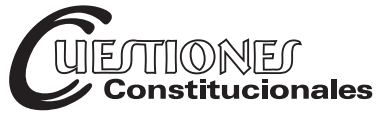

Revista Mexicana de Derecho Constitucional Núm. 44, Enero-Junio 2021

ISSN (versión electrónica): 2448-4881

\title{
El diálogo jurisprudencial entre cortes constitucionales: El caso de México
}

\author{
The judicial dialogue among constitutional courts: \\ The case of Mexico
}

Recepción: 9 de octubre de 2018

Aceptación: 16 de agosto de 2019

\author{
Josafat CORTEZ SALINAS*
}

RESUMEN: ¿Por qué las cortes supremas usan el derecho internacional? Este trabajo sugiere que en México se comenzó a utilizar dialogo jurisprudencial porque un nuevo juez con un perfil distinto (José Ramón Cossío) decidió hacerlo de forma gradual mediante su equipo de colaboradores que combinaba gente de carrera judicial y gente con trayectoria en la academia y en el litigio. Adicionalmente se muestra como el ministro capacitó a su personal en cortes y tribunales internacionales para que tuvieran cercanía y contacto con el derecho internacional. Estos factores combinados con un ambiente favorable a los derechos humanos propiciaron que la Suprema Corte comenzará a usar diálogo jurisprudencial.

Palabras clave: jueces, Suprema Corte, derecho internacional, derechos humanos.
ABSTRACT: Why do supreme courts use international law? This work suggests that, in Mexico, the arrival of a new judge with a different profile and an atypical staff progressively introduced a jurisprudential dialogue within the Court. Additionally, it is shown how the minister trained his staff in international courts and tribunals in order to get in contact with international law. These factors, combined with a favorable environment for human rights, led the Supreme Court to begin using jurisprudential dialogue.

Keywords: Judges, Supreme Court, International law, Human Rights.

* Doctor en ciencia política por la FLACSO-México. Profesor-investigador del Centro de Estudios Políticos, Facultad de Ciencias Políticas y Sociales de la UNAM. Correo electrónico: josafatcortez@politicas.unam.mx, ORCID: http://orcid.org/0000-00016754-5177. 
Esta revista forma parte del acervo de la Biblioteca Jurídica Virtual del Instituto de Investigaciones Jurídicas de la UNAM

SUMARIO: I. Introducción. II. El diálogo jurisprudencial y conducta judicial. III. La SCJN y el uso del derecho internacional. IV. Un juez y su equipo impulsando el diálogo jurisprudencial. Entrenamiento en cortes internacionales de los secretarios de Estudio y Cuenta. VI. Procesos de socialización y difusión de ideas. VII. Conclusiones. VIII. Fuentes consultadas.

\section{INTRODUCCIÓN ${ }^{1}$}

La Suprema Corte de Justicia de la Nación (SCJN) en el periodo de 2006 al 2012 citó a la Corte Interamericana 325 veces en sus sentencias, lo que la ubica en el número cuatro de América Latina que más la cita (GonzálezOcantos, 2018). ¿Por qué las cortes supremas citan a otras cortes y tribunales? ¿por qué la SCJN comenzó a utilizar las decisiones de otros tribunales internacionales antes de la reforma de 2011?

La interacción que tienen las cortes con decisiones de sus pares de otros países y tribunales internacionales por medio de citas y referencias es conocido como un "diálogo jurisprudencial" entre cortes y sentencias ejecutorias (Ahdieh, 2004). Existen distintas respuestas de por qué las cortes locales usan derecho internacional y citan a otras cortes pese a no estar obligadas a hacerlo en muchos casos. La primera es por la internacionalización del lenguaje de los derechos humanos ya que es la referencia más recurrida por las cortes supremas y la constitucionalización de los tratados internacionales. La segunda respuesta es por factores internos como motivaciones propias de los jueces y por redes y conocimiento de las decisiones de otros tribunales y por la llegada de nuevos jueces con visiones diferentes del derecho (Sandholtz, 2015; Muller y Hege, 2017; Huneeus, 2016; Gónzalez-Ocantos y Sandholtz, 2020). Una tercera respuesta se da por los mecanismos de difusión que despliega las cortes internacionales como la Corte Interamericana de su jurisprudencia (González-Ocantos, 2018).

Este trabajo estudia el caso de México como un caso menos plausible (Gerring, 2007) en el que no se espera que se cambien las decisiones de los jueces y se cite derecho internacional por tres razones: en primer lu-

1 Los datos y algunos fragmentos de este artículo fueron tomados de mi libro: Cortez (2020).

Cuestiones Constitucionales, Núm. 44, Enero-Junio 2021

ISSN: $2448-4881$ 
Esta revista forma parte del acervo de la Biblioteca Jurídica Virtual del Instituto de Investigaciones Jurídicas de la UNAM

gar, por la fuerza de las rutinas y hábitos del entrenamiento judicial de los secretarios de estudio y cuenta que permean la institución y que impedían la renovación de los criterios jurisprudenciales en la Corte (Cossio,1996). En segundo lugar, porque el formalismo-positivismo es la cultura jurídica predominante de los operadores jurídicos (Ansolabehere, 2010; Madrazo y Vela, 2011; Pérez-Perdomo, 2012). En tercer lugar, porque después de la reforma judicial de 1994 no se había consolidado una fuerte tradición entre los jueces a favor de los derechos humanos por lo que era poco probable que ocurrieran modificaciones en las decisiones de jueces y en usar derecho internacional.

Utilizamos el caso de México para comprobar como la presencia de nuevos jueces en las cortes como José Ramón Cossío -quien citó en un porcentaje importante de sus sentencias a la Corte Interamericana- (Gónzalez-Ocantos y Sandholtz, 2020) favorece el diálogo jurisprudencial, pero detallamos lo qué hace un nuevo juez: 1) en la esfera de su autonomía reorganiza su equipo de trabajo para lidiar con las rutinas de las cortes y para difundir nuevas ideas jurídicas; 2) utiliza los recursos de la Corte para capacitar a sus colaboradores en cortes y tribunales internacionales. Estos dos mecanismos explican por qué la Corte mexicana comenzó a utilizar antes de la reforma de 2011 diálogo jurisprudencial. Para respaldar el argumento se realizaron entrevistas con operadores jurídicos claves en la Suprema Corte de Justicia sobre los procesos de socialización y las fuentes que se utilizan en la construcción de las sentencias.

\section{EL DIÁLOGO JURISPRUDENCIAL Y LA CONDUCTA JUDICIAL}

El uso del derecho internacional por las cortes y tribunales se ha acelerado en los últimos (Bahdi, 2002). En América Latina es reciente el análisis sobre el uso que hacen las cortes del derecho internacional y cómo usan la literatura jurídica en las sentencias, sin embargo, cada vez se comienzan a tener más trabajos que abordan sobre todo la interacción de las cortes locales con la Corte Interamericana de Derechos Humanos (Rodiles, 2016; Mohallem, 2017; González-Ocantos, 2018; Gónzalez-Ocantos y Sandholtz, 2020).

Una de las formas de analizar la conducta judicial es prestar atención a la forma en la que se construyen las sentencias judiciales. Estudiar quiénes las hacen y cómo las elaboran permite reconocer pautas y formas en 
Esta revista forma parte del acervo de la Biblioteca Jurídica Virtual del Instituto de Investigaciones Jurídicas de la UNAM

las que el juez concibe su función judicial. Los estudios sobre comportamiento judicial desde la ciencia política latinoamericana recientemente han prestado atención a analizar la influencia de las ideas jurídicas en las decisiones judiciales (González-Ocantos, 2016; Matthew, 2016). Sin embargo, una tarea pendiente es explicar cómo se utiliza el derecho por parte de los jueces y una de las formas de hacerlo es mediante el análisis de las fuentes que utiliza para construir la decisión judicial como un indicador de cambios en la cultura jurídica de las cortes.

El punto de partida es precisar cuál es incentivo que tienen los jueces en utilizar determinadas fuentes en sus sentencias. Desde la propuesta que presento uno de los motivos de los jueces es el deseo de controlar cómo los perciben e influir sobre los otros y su visión del mundo (Devins y Baum, 2019). La audiencia en la que los ministros están más pendientes es la de las elites legales porque son su círculo más cercano y el que les interesa influir sobre cómo perciben que realizan su trabajo jurisdiccional (Devins y Baum, 2019). Buscan maximizar determinado prestigio y satisfacción por el trabajo que realizan y por eso utilizan el derecho de manera instrumental (Lax, 2011).

El segundo incentivo es que los jueces también son abogados que estudiaron derecho y aprendieron una forma de entenderlo y la forma en la que se deben de resolver las disputas jurídicas. Las citas en las sentencias permiten explicar la influencia de las ideas jurídicas como una restricción interna (Gillman, 2001). Cuando los jueces y sus secretarios escriben las sentencias expresan la forma en la que conciben el derecho y la forma en la que lo aprendieron en las escuelas de derecho y sus posgrados, así como por sus procesos de socialización que varían entre cada actor judicial. Por medio de las citas aprendemos la forma en la que usan el derecho constitucional y de dónde vienen sus ideas jurídicas o siguiendo a GonzálezOcantos (2016) sus preferencias legales.

Las nuevas ideas jurídicas y formas de entender el derecho constitucional como el diálogo jurisprudencial y el derecho comparado son los temas que están en el centro del debate constitucional y político. Innovar en estas ideas permite al juez: a) construir una reputación y prestigio en una comunidad externa como los sectores académicos y b) mostrar su visión del derecho y la forma en la que aprendieron la forma de resolver los problemas jurídicos (Cortez, 2020). 
Esta revista forma parte del acervo de la Biblioteca Jurídica Virtual del Instituto de Investigaciones Jurídicas de la UNAM

Este trabajo sostiene que uno de los factores que genera el diálogo jurisprudencial son las motivaciones personales de los jueces, en específico de aquellos con una mirada diferente del derecho. La literatura ha comprobado que cuando llegan nuevos jueces a las cortes con una nueva cultura jurídica las decisiones en materia de derechos humanos comienzan a cambiar (Couso, 2010; Couso y Hilbink, 2010; Nunes, 2010). Sin embargo, esta hipótesis no específica el mecanismo que despliegan los nuevos jueces para incidir en las cortes y romper rutinas. Mi argumento señala que los jueces con una visión diferente del derecho para poder incidir en las decisiones y construir un prestigio lo primero que hacen es reorientar su equipo de trabajo porque es el primer recurso con el que cuentan. Reconfigurar el perfil de sus colaboradores y la forma en la que trabajaban es lo que se puede hacer desde los márgenes para impulsar un cambio gradual en las cortes (Cortez, 2020).

El juez con un equipo novedoso va a desplegar sus estrategias de cambio y una de ellas va a ser impulsar el diálogo jurisprudencial mediante su equipo de colaboradores. Con este propósito va a utilizar los recursos de la Corte y va a mandar a sus colaboradores a cursos y estancias tanto en la Corte Interamericana de Derechos Humanos en Costa Rica como en otras cortes del mundo. La capacitación va a fortalecer el conocimiento de los casos y las decisiones. Un factor fundamental en la región es que los jueces no siempre conocen el derecho internacional o la jurisprudencia de los tribunales internacionales lo que dificulta que lo plasmen en sus sentencias en materia de derechos humanos (González-Ocantos, 2016). Una condición necesaria es conocer el derecho internacional y después viene el problema de operacionalizarlo en las sentencias judiciales y buscar la aceptación en las cortes y en los tribunales inferiores. Por eso el juez primero los manda a capacitar para que conozcan el derecho internacional (Cortez, 2020).

Los jueces no trabajan solos, sino que necesitan un equipo de colaboradores que los respalde en sus objetivos y decisiones. Los secretarios de estudio y cuenta o letrados son sus aliados y son los que operacionalizan las ideas jurídicas y utilizan el diálogo jurisprudencial porque lo aprenden en sus procesos de socialización en las escuelas de derecho y en sus posgrados en los cursos de capacitación que cursan (Cortez, 2019). Los secretarios estudiaron derecho y tienen determinadas preferencias legales (González-Ocantos, 2016) y utilizan el derecho como un estado de la men- 
Esta revista forma parte del acervo de la Biblioteca Jurídica Virtual del Instituto de Investigaciones Jurídicas de la UNAM

te, es una restricción interna en su conducta que les indica la forma en la que se deben resolver los problemas jurídicos (Gillman, 2001).

Por eso, el primer paso de los jueces es reorientar su equipo de trabajo buscando determinados perfiles y después capacitarlos en diferentes cortes en el mundo. En el caso de México en el año 2017 todos los secretarios de Estudio y Cuenta que tienen doctorado lo estudiaron en España (Cortez, 2019), eso podría ser indicativo de por qué citan el Tribunal Constitucional Español más que otros tribunales.

\section{LA SCJN Y EL USO DEL DERECHO INTERNACIONAL}

En el debate sobre la relación de la SCJN con el derecho internacional existían dos posturas encontradas. La mirada nacionalista apela al principio de soberanía nacional y de la pirámide jerárquica en la cual está la Constitución por encima de cualquier norma (Serna, 2012). Por otro lado, está la mirada que reconoce la importancia de los tratados internacionales y de la Corte Interamericana, y la necesidad de contar con una defensa supranacional de los derechos humanos (Serna, 2012).

En este sentido, el artículo 133 constitucional que trata sobre la jerarquía de los tratados internacionales en el ordenamiento mexicano no ha sido modificado desde 1934; se ha mantenido constante desde entonces, pero lo que sí ha variado es la interpretación que hacen los jueces. El tema de fondo de la disputa jurídica es determinar el papel que tienen los tratados internacionales y su vínculo jerárquico con la Constitución.

Tres decisiones han marcado el debate en esta materia. La primera fue en 1992 en un contexto autoritario y una Corte sin independencia. En la tesis P.C/1992 se sostuvo que los tratados internacionales estaban en el mismo nivel normativo que las leyes federales y por debajo de la Constitución.

En 1999 una Suprema Corte de Justicia renovada en su independencia y poder, cambió el criterio de interpretación del artículo 133 constitucional, en el amparo 1475/1998 determinó que los tratados internacionales están ubicados por encima de las leyes federales y debajo de la Constitución. En 2007 la Corte volvió a decidir sobre el significado del artículo 133 constitucional. En esta ocasión mediante la tesis jurisprudencial P IX/2007 la Corte sostuvo que los tratados internacionales se ubican por encima de las leyes generales, federales y locales.

Cuestiones Constitucionales, Núm. 44, Enero-Junio 2021

ISSN: 2448-4881 
Esta revista forma parte del acervo de la Biblioteca Jurídica Virtual del Instituto de Investigaciones Jurídicas de la UNAM

Desde entonces distintos actores han apelado a que la Corte definiera mediante la interpretación del artículo 133 constitucional la jerarquía de los tratados internacionales. Fue hasta la reforma en materia de derechos humanos con las adiciones al artículo 1 constitucional en la que existió más claridad en el tema.

La reforma constitucional en derechos humanos de 2011 amplió las fuentes normativas de los derechos humanos: la Constitución y los tratados internacionales, a los que se presupone que los jueces en sus decisiones tienen que apegarse (Castilla, 2011). Uno de los rasgos que más sobresalen es el que los tratados internacionales, en materia de derechos humanos, tienen estatura constitucional. El artículo 1 también indica: "Las normas relativas a los derechos humanos se interpretarán de conformidad con esta Constitución y con los tratados internacionales de la materia favoreciendo en todo tiempo a las personas la protección más amplia." Se estipuló como obligación del Estado garantizar y proteger los derechos humanos a través de garantías y mecanismos. Los derechos humanos se colocan en el centro del ordenamiento jurídico como límites al ejercicio del poder político y columna vertebral de la constitución política. Pese a la claridad de la reforma la SCJN se ha tenido que pronunciar sobre los alcances del artículo 1o. constitucional en la Contradicción de Tesis 293/2011. Después de un largo proceso de discusión entre los jueces se asentó entre otras cosas que los derechos humanos consagrados en tratados internacionales tienen rango constitucional. La contradicción de tesis sugiere que pese a los cambios legales el comportamiento de los jueces varia sobre los alcances de los artículos constitucionales. La reforma en derechos humanos aceleró decisiones que garantizan derechos humanos y el diálogo jurisprudencial e implicó que el juez debe aprender una nueva forma de entender el derecho y sus fuentes en su labor interpretativa. Por mencionar un ejemplo, el juez debe considerar los derechos estipulados en la Convención Americana y más de 50 tratados en materia de Derechos Humanos firmados por el México (Castilla, 2011).

\section{UN JUEZ Y SU EQUIPO IMPULSANDO EL DIÁLOGO JURISPRUDENCIAL}

Los jueces están rodeados por un equipo de trabajo que los respalda. El primer recurso con el que cuentan los jueces cuando ingresan a la Corte es la posibilidad de conformar y seleccionar a sus colaboradores. Es su 
Esta revista forma parte del acervo de la Biblioteca Jurídica Virtual del Instituto de Investigaciones Jurídicas de la UNAM

esfera de autonomía en la que de acuerdo con sus objetivos eligen diferentes perfiles y trayectorias profesionales y académicas (Cortez, 2020). Los jueces delegan la elaboración de las sentencias y del trabajo jurisdiccional en un equipo de abogados con diferentes trayectorias profesionales y habilidades.

Los secretarios de estudio y cuenta cumplen con tres funciones: incorporan distintas miradas a los problemas jurídicos, reducen costos de aprendizaje de las rutinas de las cortes y son fuentes de información que permiten difundir nuevas ideas jurídicas (Cortez, 2019: 219-236). Por eso es tan importante analizar la estructura de las ponencias de los jueces y el perfil de su personal. Los jueces necesitan aliados que conozcan derecho internacional y que los apoyen en sus propósitos y en la difusión de ideas jurídicas.

En el caso de México, José Ramón Cossío llegó a la SCJN en 2003 y su trayectoria profesional se distanciaba del modelo tradicional del juez de la corte porque provenía de la academia y había estudiado la historia del Poder Judicial y su comportamiento en la historia política del país. José Ramón Cossío es el actor que va a influir de forma gradual en la SCJN mediante la conformación de un equipo de trabajo novedoso que va a combinar abogados de carrera judicial con abogados con experiencia en el litigio y la academia. Su equipo de trabajo fue innovador en ese momento en la SCJN y le permitía navegar en los hábitos y rutinas de la corte para poder innovar y difundir nuevas ideas jurídicas (Cortez, 2020).

Después de construir su equipo de colaboradores el juez estableció dinámicas de trabajo en conjunto entre secretarios externos al Poder Judicial y gente de carrera judicial. En las entrevistas realizadas se aprecia como el juez Cossío puso énfasis en su ponencia de usar derecho comparado y en usar literatura jurídica, desde antes de la reforma en derechos humanos en 2011 que señalaba que los tratados internacionales y las sentencias de la Corte IDH estaban al mismo nivel que la Constitución.

De acuerdo con los operadores jurídicos había una directriz del ministro de usar diálogo jurisprudencial en la medida de lo posible en los proyectos de sentencias porque sabían que los ministros de carrera judicial no consideraban adecuado citar otras fuentes de derecho. Tenía que construirse de forma gradual, uno de los operadores jurídicos sostiene: "Nos moderamos porque sabíamos que sólo generaríamos rechazo innecesario, 
Esta revista forma parte del acervo de la Biblioteca Jurídica Virtual del Instituto de Investigaciones Jurídicas de la UNAM

que teníamos que ir como incrementalmente". ${ }^{2}$ Para romper con una institución que se resiste a incorporar el diálogo jurisprudencial el ministro Cossío y su equipo fueron poco a poco en el uso de derecho internacional.

De acuerdo con las entrevistas realizadas el ministro Cossío contrató a alguien que apoyara a la ponencia y a sus secretarios de estudio y cuenta en el uso de derecho internacional. El abogado Karlos Castilla, egresado de la UNAM, trabajó en una etapa en la ponencia con la tarea de apoyar a los secretarios de forma sistematizada en el uso del diálogo jurisprudencial.

Antes de la reforma en derechos humanos del 2011 la ponencia de Cossío estaba en un diálogo con otras cortes y con otras fuentes de literatura. Dice un operador jurídico sobre citar CIDH y otros tribunales internacionales.

El verdadero mérito era hacer esto cuando había tanta resistencia, porque ... después del 11 de junio de 2011 era absurdo no hacerlo, porque en la Constitución ya era evidente o es exageradamente clara en decir, derechos humanos y decir, derechos reconocidos en tratados obligados, y antes de eso tenías el 133 y tenías que construir a partir del $133 .^{3}$

El diálogo jurisprudencial apareció en la SCJN de forma gradual y paulatina impulsado por un juez. Su ancla era la interpretación del 133 constitucional para poder incorporar otras fuentes de derecho como tratados internacionales o CIDH. Por ejemplo, las referencias en los proyectos de sentencia en materia de libertad de expresión, matrimonio igualitario y aborto en un periodo de 1995-2015 son variadas: CIDH, Corte Suprema de Estados Unidos, Tribunal Constitucional Español, Tribunal Europeo de Derechos Humanos, Corte Constitucional de Colombia y Corte Sudafricana (Cortez, 2020).

2 Entrevista realizada en la Ciudad de México, 20 de septiembre de 2016.

3 Entrevista en la Ciudad de México. 24 de agosto de 2016. 
Esta revista forma parte del acervo de la Biblioteca Jurídica Virtual del Instituto de Investigaciones Jurídicas de la UNAM

\section{ENTRENAMIENTO EN CORTES INTERNACIONALES DE LOS SECRETARIOS DE ESTUDIO Y CUENTA}

El ministro Cossío al llegar a la SCJN modificó su equipo de trabajo y buscó lidiar con los hábitos y rutinas de la institución, pero también quería difundir nuevas ideas jurídicas y formas de construir las sentencias judiciales. Una vez que cambió su equipo de trabajo el juez capacitó a su personal en derecho internacional usando los recursos de la corte para que sus secretarios realizaran estancias en cortes y tribunales internacionales. No basta con tener secretarios con experiencia en la academia y en el litigio, ni un abogado que los apoye en el uso del derecho internacional, sino que también es necesario capacitar al personal con estancias en otros tribunales para aprender su forma de trabajo y conocer la jurisprudencia. El ministro buscó que su personal se capacitará en esos temas.

Secretarios de Estudio y Cuenta de la ponencia del juez José Ramón Cossío que asistieron a cursos y estancias

\begin{tabular}{|c|c|c|c|}
\hline Secretario & $A \tilde{n} o$ & Lugar de curso o capacitación & Título del reporte de trabajo \\
\hline Francisca Pou & 2005 & CIDH. Costa Rica & \\
\hline $\begin{array}{l}\text { Laura Patricia } \\
\text { Rojas }\end{array}$ & 2005 & CIDH. Costa Rica & \\
\hline $\begin{array}{l}\text { Rosalba } \\
\text { Rodríguez }\end{array}$ & 2005 & CIDH. Costa Rica & \\
\hline Francisca Pou & 2006 & $\begin{array}{l}\text { Suprema Corte de Justicia de } \\
\text { Colombia }\end{array}$ & \\
\hline $\begin{array}{l}\text { Lorena Gos- } \\
\text { linga }\end{array}$ & 2006 & CIDH. Costa Rica & $\begin{array}{l}\text { "Evolución del Sistema } \\
\text { Interamericano de Protección } \\
\text { y Promoción de los Derechos } \\
\text { Humanos" }\end{array}$ \\
\hline $\begin{array}{l}\text { Dolores } \\
\text { Rueda }\end{array}$ & 2006 & $\mathrm{CIDH}$ & $\begin{array}{l}\text { "El fortalecimiento del Sistema } \\
\text { Regional de Protección de los } \\
\text { Derechos Humanos en Latino } \\
\text { América" }\end{array}$ \\
\hline Francisca Pou & 2008 & Universidad de Toronto. & \\
\hline
\end{tabular}


Esta revista forma parte del acervo de la Biblioteca Jurídica Virtual del Instituto de Investigaciones Jurídicas de la UNAM

\begin{tabular}{|c|c|c|c|}
\hline $\begin{array}{l}\text { Laura Patricia } \\
\text { Rojas }\end{array}$ & 2008 & $\begin{array}{l}\text { Corte Constitucional de Co- } \\
\text { lombia }\end{array}$ & $\begin{array}{l}\text { "Modulación de los efectos } \\
\text { temporales de las sentencias de } \\
\text { inexequibilidad dictadas por la } \\
\text { Corte Constitucional Colom- } \\
\text { biana." }\end{array}$ \\
\hline \begin{tabular}{l|} 
Lorena Gos- \\
linda Ramírez
\end{tabular} & 2008 & $\begin{array}{l}\text { Facultad de Derecho. Universi- } \\
\text { dad de Toronto }\end{array}$ & $\begin{array}{l}\text { Derecho, Ciencia y Tecnología } \\
\text { en la Suprema Corte Canadien- } \\
\text { se: estándares para la admi- } \\
\text { sión de evidencia científica y } \\
\text { técnica." }\end{array}$ \\
\hline Roberto Lara & 2008 & CIDH. Costa Rica. & $\begin{array}{l}\text { Derechos Humanos en el Sis- } \\
\text { tema Interamericano: un caso } \\
\text { paradigmático }\end{array}$ \\
\hline Francisca Pou & 2009 & $\begin{array}{l}\text { Relatoría Especial para la Li- } \\
\text { bertad de Expresión. Comisión } \\
\text { Interamericana de Derechos } \\
\text { Humanos. Organización de los } \\
\text { Estados Americanos. }\end{array}$ & $\begin{array}{l}\text { La libertad de expresión en } \\
\text { América Latina }\end{array}$ \\
\hline Francisca Pou & 2009 & $\begin{array}{l}\text { Paraguay. Seminario en Latino- } \\
\text { américa de Teoría Constitucio- } \\
\text { nal y Política (SELA) }\end{array}$ & $\begin{array}{l}\text { "Derecho y sexualidad, el papel } \\
\text { de la Iglesia" }\end{array}$ \\
\hline Roberto Lara & 2009 & $\begin{array}{l}\text { Paraguay. Seminario en Latino- } \\
\text { américa de Teoría Constitucio- } \\
\text { nal y Política (SELA) }\end{array}$ & $\begin{array}{l}\text { Reporte de trabajo derivado del } \\
\text { Seminario en Latinoamérica de } \\
\text { Teoría Constitucional y Política } \\
\text { Celebrado en la Ciudad de } \\
\text { Asunción, Paraguay" }\end{array}$ \\
\hline Roberto Lara & 2009 & $\begin{array}{l}\text { Tribunal Constitucional de } \\
\text { España }\end{array}$ & $\begin{array}{l}\text { Competencias y principios: } \\
\text { una relación incomoda, pero } \\
\text { necesaria }\end{array}$ \\
\hline $\begin{array}{l}\text { Juan Carlos } \\
\text { Roa }\end{array}$ & 2009 & $\begin{array}{l}\text { Canadá-Vancouver. Congreso } \\
\text { de la "International Fiscal } \\
\text { Association", denominado pre- } \\
\text { cisamente "Tax amnesties" }\end{array}$ & $\begin{array}{l}\text { Sobre las denominadas "amnis- } \\
\text { tías fiscales" } \\
\text { como método alternativo para } \\
\text { la extinción de la deuda tribu- } \\
\text { taria, y los efectos que estás } \\
\text { tienen en la distribución } \\
\text { de las cargas fiscales entre los } \\
\text { gobernados. }\end{array}$ \\
\hline $\begin{array}{l}\text { Lorena Gos- } \\
\text { linga }\end{array}$ & 2009 & $\begin{array}{l}\text { Departamento de Derecho de } \\
\text { la Universidad Pompeu Fabra. } \\
\text { España }\end{array}$ & Hostigamiento y acoso sexual. \\
\hline
\end{tabular}


Esta revista forma parte del acervo de la Biblioteca Jurídica Virtual del Instituto de Investigaciones Jurídicas de la UNAM

\begin{tabular}{|c|c|c|c|}
\hline $\begin{array}{l}\text { Juan Carlos } \\
\text { Roa }\end{array}$ & 2010 & $\begin{array}{l}\text { 64o. Congreso de la "Inter- } \\
\text { national Fiscal Association" } \\
\text { ("IFA"), celebrado en agosto } \\
\text { y septiembre de } 2010 \text { en la } \\
\text { Ciudad de Roma, }\end{array}$ & $\begin{array}{l}\text { El desgaste en las relaciones } \\
\text { entre fisco y contribuyentes, y } \\
\text { las alternativas para optimizar- } \\
\text { las, en el marco de la Organi- } \\
\text { zación para la Cooperación y el } \\
\text { Desarrollo Económico. }\end{array}$ \\
\hline Roberto Lara & 2010 & $\begin{array}{l}\text { Tribunal Supremo y la Audien- } \\
\text { cia Nacional de España, }\end{array}$ & $\begin{array}{l}\text { La motivación de los hechos } \\
\text { en los casos de "convicción } \\
\text { incompleta" }\end{array}$ \\
\hline $\begin{array}{l}\text { Karlos Cas- } \\
\text { tilla }\end{array}$ & 2011 & $\begin{array}{l}\text { Tribunal Constitucional de } \\
\text { España }\end{array}$ & $\begin{array}{l}\text { "La interpretación conforme a } \\
\text { tratados de derechos humanos. } \\
\text { Una mirada a la experiencia } \\
\text { española para el futuro de } \\
\text { México" }\end{array}$ \\
\hline Roberto Lara & 2012 & $\begin{array}{l}\text { XIV Seminario Internacional de } \\
\text { Teoría del Derecho. Universi- } \\
\text { dad Nacional del Sur-Argentina }\end{array}$ & $\begin{array}{l}\text { "La salvaguarda del derecho a } \\
\text { la no discriminación a través de } \\
\text { la sanción" }\end{array}$ \\
\hline $\begin{array}{l}\text { Karlos Cas- } \\
\text { tilla }\end{array}$ & & $\begin{array}{l}\text { Suprema Corte de Justicia de } \\
\text { Colombia. }\end{array}$ & $\begin{array}{l}\text { Otra Manera de hacer justi- } \\
\text { cia. La Corte Constitucional } \\
\text { Colombiana }\end{array}$ \\
\hline $\begin{array}{l}\text { Juan Carlos } \\
\text { Roa }\end{array}$ & & $\begin{array}{l}65 \text { congreso Mundial de In- } \\
\text { ternational Fiscal Association. } \\
\text { París-Francia }\end{array}$ & \\
\hline $\begin{array}{l}\text { Dolores } \\
\text { Rueda }\end{array}$ & & $\mathrm{CIDH}$ & $\begin{array}{l}\text { El fortalecimiento del Sistema } \\
\text { Regional de Protección de los } \\
\text { Derechos Humanos en Latino } \\
\text { América" }\end{array}$ \\
\hline $\begin{array}{l}\text { Carmen Ver- } \\
\text { gara López }\end{array}$ & & $\mathrm{CIDH}$ & $\begin{array}{l}\text { Análisis del Sistema Interame- } \\
\text { ricano de Derechos Humanos }\end{array}$ \\
\hline \begin{tabular}{|l|} 
Rosalba \\
Rodríguez \\
Mireles \\
\end{tabular} & & $\begin{array}{l}\text { Corte Constitucional de Co- } \\
\text { lombia }\end{array}$ & $\begin{array}{l}\text { "Capacitación in situ en la Cor- } \\
\text { te Constitucional de Colombia, } \\
\text { con sede en Bogotá" }\end{array}$ \\
\hline $\begin{array}{l}\text { Laura Patricia } \\
\text { Rojas. }\end{array}$ & & Costa Rica. & $\begin{array}{l}\text { "Omisión legislativa. Estudio } \\
\text { comparativo de su tratamiento } \\
\text { y forma de control entre la Sala } \\
\text { Constitucional de Costa Rica } \\
\text { y la Suprema Corte de Justicia } \\
\text { Mexicana" }\end{array}$ \\
\hline $\begin{array}{l}\text { Dolores } \\
\text { Rueda }\end{array}$ & & $\begin{array}{l}\text { Suprema Corte de Justicia de } \\
\text { Colombia }\end{array}$ & $\begin{array}{l}\text { "El bloque de constitucionali- } \\
\text { dad en el sistema colombiano" }\end{array}$ \\
\hline $\begin{array}{l}\text { Carmen Ver- } \\
\text { gara López }\end{array}$ & & $\begin{array}{l}\text { Suprema Corte de Justicia } \\
\text { Colombia }\end{array}$ & $\begin{array}{l}\text { "Ponderación entre derechos } \\
\text { fundamentales" }\end{array}$ \\
\hline
\end{tabular}

Cuestiones Constitucionales, Núm. 44, Enero-Junio 2021

ISSN: $2448-4881$ 
Esta revista forma parte del acervo de la Biblioteca Jurídica Virtual del Instituto de Investigaciones Jurídicas de la UNAM

\begin{tabular}{|l|l|l|l|}
\hline $\begin{array}{l}\text { Juan Carlos } \\
\text { Roa }\end{array}$ & Estados Unidos & $\begin{array}{l}\text { Algunos acercamientos al } \\
\text { control de la elusión fiscal en la } \\
\text { jurisprudencia norteamericana. }\end{array}$ \\
\hline $\begin{array}{l}\text { Dolores } \\
\text { Rueda }\end{array}$ & España. & $\begin{array}{l}\text { "La responsabilidad patrimonial } \\
\text { del estado: México y España" }\end{array}$ \\
\hline $\begin{array}{l}\text { Carmen Ver- } \\
\text { gara López }\end{array}$ & España & $\begin{array}{l}\text { "Seguridad social, división } \\
\text { jurisdiccional de la seguridad } \\
\text { social. España y México" }\end{array}$ \\
\hline
\end{tabular}

FUENTE: Elaboración propia con los datos del libro Cortez (2020).

La participación de integrantes de la ponencia, tanto internos como externos, en cursos de capacitación y congresos se mantiene constante. Es importante resaltar que desde antes de la reforma de 2011 en derechos humanos los secretarios de estudio y cuenta de la ponencia de Cossío ya estaban en Costa Rica tomando cursos y viendo el funcionamiento de la CIDH. También sobresale la presencia de los secretarios en Colombia en la Corte Constitucional y en el Tribunal Constitucional Español. Tanto los abogados de carrera judicial como los externos se vieron beneficiados con estas becas para aprender y ver lo que estaban haciendo en otras partes del mundo. El contacto con otras cortes y jueces permite relacionarse y socializar formas de entender el derecho y la resolución de problemas jurídicos.

Había un reconocimiento de la necesidad de conocer lo que estaba diciendo en materia de derechos humanos la CIDH y saber cómo estaban resolviendo problemas parecidos en otras partes del mundo. Si el juez constitucional no conoce una cultura jurídica distinta su círculo cercano pueden aprender y capacitarse sobre lo que está sucediendo en otras cortes. Estos cursos favorecieron el diálogo jurisprudencial en las sentencias de la SCJN.

\section{PROCESOS DE SOCIALIZACIÓN Y DIFUSIÓN DE IDEAS}

Los jueces y sus colaboradores son portadores de ideas jurídicas que plasman cuando escriben las sentencias judiciales. Como abogados también están expuestos a un ambiente y a un contexto de interacciones como procesos de socialización (Cortez, 2020). En el caso de México la directriz de usar derecho internacional venía del ministro, pero de acuerdo con las entrevistas también era una forma de entender el derecho por parte de los 
Esta revista forma parte del acervo de la Biblioteca Jurídica Virtual del Instituto de Investigaciones Jurídicas de la UNAM

secretarios de estudio y cuenta; es decir, había marcos cognitivos compartidos que facilitaban el uso del derecho internacional. En las entrevistas realizadas un amplio porcentaje de los secretarios de estudio y cuenta se muestra favorable al uso de derecho comparado y al uso de literatura jurídica en las sentencias. Su visión del derecho constitucional y sus marcos cognitivos les marca pautas de comportamiento distintas y mayor apertura al diálogo jurisprudencial.

Después de la ponencia de Cossío en otros proyectos de otros jueces comienzan a aparecen referencias a decisiones de otras cortes y tribunales. ¿Por qué los jueces usan determinadas decisiones de unos tribunales y no de otros? Tiene que ver con que los jueces, así como sus secretarios de estudio y cuenta son portadores de ideas jurídicas, y la forma en la que usan determinadas fuentes del derecho se relaciona con sus espacios de socialización y de estudio (Cortez, 2020); por ejemplo, uno de los operadores jurídicos afirma sobre las fuentes a las que recurre:

... yo me siento muy cómodo con los precedentes americanos; porque son también precedentes muy cortos, son de fácil acceso y comúnmente son precedentes que marcan la discusión en preguntas generales, en preguntas jurídicas. ¿Tiene o no un derecho a abortar esta persona?, entonces como se hacen preguntas generales es bastante útil acudir a los precedentes americanos. ${ }^{4}$

Otro de los operadores afirma la importancia de ver lo que hacen otras cortes y tribunales cuando no existen precedentes claros en la SCJN

siempre consultamos derecho comparado porque puedes ir a tribunales que tienen mucha más experiencia y mucha más trayectoria y te sirve de referencia para ver estándares y ver como los están haciendo. Y bueno, a mí me parece utilísimo. Nosotros en todos los asuntos difíciles siempre lo hemos hecho, sobre todo en este tipo de casos que son más complicados, en los que no hay como un presente claro aquí. ${ }^{5}$

El uso del diálogo jurisprudencial se relaciona con la socialización, los espacios de trabajo y la forma de entender el derecho. Los secretarios también actúan de forma sincera al usar el derecho como un 'estado de la

4 Entrevista en la Ciudad de México 10 de mayo de 2016.

5 Entrevista en la Ciudad de México. 1o. de septiembre de 2016.

Cuestiones Constitucionales, Núm. 44, Enero-Junio 2021

ISSN: $2448-4881$ 
Esta revista forma parte del acervo de la Biblioteca Jurídica Virtual del Instituto de Investigaciones Jurídicas de la UNAM

mente' (Gillman, 2001). Cuando elaboran los proyectos de sentencia son congruentes con los marcos cognitivos adquiridos durante sus trayectorias. Un operador jurídico dice: "Mis primeros referentes están en el sistema interamericano, porque los conozco, y ni siquiera es que los busque, lo sé. Entonces, pues el sistema interamericano está muy presente". ${ }^{6}$

Después de la reforma de derechos humanos en 2011, el uso de tratados internacionales y las sentencias de la CIDH aparecen con mayor énfasis en los proyectos de sentencias de todos los jueces de la SCJN. Sin embargo, pese a que un amplio porcentaje de los entrevistados está a favor de usar otras decisiones de otros tribunales también aparece el tema de la metodología para usar estas fuentes de forma sistematizada. "Creo que, desafortunadamente no hemos logrado tener una metodología. Creo que es un poco dependiente del secretario que proyecte".

Me parece importante resaltar dos ideas sobre la difusión de ideas en la Corte. La primera es que parece que no existe un método para acercarse a las decisiones de las demás cortes, es decir, los jueces y secretarios tienen toda la libertad de citar las decisiones que consideren más adecuadas para justificas sus decisiones. En el caso de México, el ministro Cossío orientó su ponencia hacia el diálogo jurisprudencial por prestigio y reputación y por estar cerca de una comunidad académica. La segunda, es que las fuentes que se utilizan dependen del secretario que proyecta las sentencias, sus estudios y su trayectoria profesional. Los secretarios son agentes portadores de ideas jurídicas y los jueces los utilizan para actualizarse y debatir ideas jurídicas.

\section{CONCLUSIONES}

Este trabajo se inserta en la discusión de factores internos y motivaciones de los jueces y sostiene que las cortes usan derecho internacional cuando un juez decide hacerlo para obtener prestigio y difundir nuevas ideas jurídicas. Para lograrlo se rodea de colaboradores con trayectorias académicas y profesionales distintas a las tradicionales. Una vez que tiene a su equipo de abogados el juez decide capacitarlos y entrenarlos en cortes internacionales. Son dos mecanismos entrelazados. El primero es que el juez estruc-

6 Entrevista en la Ciudad de México. 17 de agosto de 2016.

7 Entrevista en la Ciudad de México. 5 de septiembre de 2016. 
Esta revista forma parte del acervo de la Biblioteca Jurídica Virtual del Instituto de Investigaciones Jurídicas de la UNAM

tura un nuevo equipo de trabajo y los manda a capacitar y entrenar en tribunales y cortes internacionales. El segundo es que los secretarios cuando escriben sus sentencias muestran su visión del derecho que aprendieron en sus escuelas, posgrados y en sus procesos de socialización.

La ponencia de José Ramón Cossío estaba en un diálogo jurisprudencial antes del caso Radilla y de la reforma en materia de derechos humanos de 2011 por tener un equipo de trabajo novedoso y porque capacito a sus colaboradores. Un factor fundamental para el uso de diálogo jurisprudencial son los secretarios de estudio y cuenta y sus procesos de socialización porque ellos operacionalizan las ideas jurídicas. Si el juez no conoce el derecho internacional o quiere impulsar su uso sus mejores aliados son los secretarios que le apoyan en la construcción de las sentencias. Es necesario que la literatura jurídica y politológica les preste atención a los secretarios de estudio y cuenta como uno de los factores que puede explicar el comportamiento judicial.

\section{FUENTES CONSULTADAS}

AHDieH, R. (2004). Between Dialogue and Decree: International Review of National Courts. New York University Law Review. 79 (6).

Ansolabehere, K. (2010). More Power, More Rights? The Supreme Court and Society in Mexico. En Couso, J. et al. (eds.). Cultures of Legality: Judicialization and Political Activism in Latin America. Cambridge: Cambridge University Press.

BAHDI, R. (2002). Globalization of Judgment: Transjudicialism and the Five Faces of International Law in Domestic Courts. The George Washington International Law Review. 34 (3).

CASTILla, K. (2011). Un nuevo panorama constitucional para el Derecho Internacional de los derechos humanos en México. Estudios Constitucionales. 9 (2).

CorteZ, J. (2020). Ideas, innovación y cambio organizacional en la Suprema Corte de Justicia de la Nación. México: UNAM, Instituto de Investigaciones Jurídicas-Facultad de Ciencias Políticas y Sociales.

Cortez, J. (2019). Secretarios de estudio y cuenta en la Suprema Corte de México: un actor esencial pero olvidado. Política y Gobierno, XXVI 
Esta revista forma parte del acervo de la Biblioteca Jurídica Virtual del Instituto de Investigaciones Jurídicas de la UNAM

(2). Disponible en: http://www.politicaygobierno.cide.edu/index.php/ pyg/article/view/1299/984.

Cossío, J. (1996). Cuadernos para la reforma judicial: jurisdicción federal y carrera judicial en México. México: UNAM, Instituto de Investigaciones Jurídicas.

Couso, J. (2010). The Transformation of Constitutional Discourse and the Judicialization of Politics in Latin America. En Couso, J. et al. Cultures of Legality: Judicialization and Political Activism in Latin America. Nueva York: Cambridge University Press.

Couso, J. (2010). "The Transformation of Constitutional Discourse and the Judicialization of Politics in Latin America". En Couso, J. et al. (eds.). Cultures of Legality: Judicialization and Political Activism in Latin America. Cambridge: Cambridge University Press. Disponible en: doi:10.1017/CBO9780511730269.006.

Couso, J. y HiLbinK, L. (2010). Del quietismo al activismo incipiente: las raíces institucionales e ideológicas de la defensa de los derechos en Chile. En Helmke, G. y Ríos FigueroA, J. (eds.). Tribunales constitucionales en América Latina, México. México: Poder Judicial de la Federación.

Devins, N. y Baum, L. (2019). The Company they Keep. How Partisan Divisions to the Supreme Court. New York: Oxford University Press. Disponible en: https://doi.org/10.1017/S1537592719001890.

Gerring, J. (2007). Case Study Research: Principles and Practices. Nueva York: Cambridge University Press.

GonZÁLEZ-OCANTOS, E. (2018). Communicative Entrepreneurs: The Case of the Inter-American Court of Human Rights' Dialogue with National Judges. International Studies Quartely, 62 (4), 737-750. Disponible en: https://doi.org/10.1093/isq/sqy040.

GonZÁlez-OcAntos, E. (2016). Shifting Legal Visions: Judicial Change and Human Rights Trials in Latin America, Nueva York, Cambridge University Press. Disponible en: https://doi.org/10.1017/ CBO9781316535509.

GonZÁlez-OcAntos, E. y SANDhOltz, Wayne (2020). Constructing a Regional Human Rights Legal Order: The Inter-American Court, National Courts, and Judicial Dialogue, 1988-2014. International Journal of Constitutional Law. 
Esta revista forma parte del acervo de la Biblioteca Jurídica Virtual del Instituto de Investigaciones Jurídicas de la UNAM

Madrazo, A. y Vela, E. (2011). The Mexican Supreme Court's (Sexual) Revolution? Texas Law Review, 89, (7).

Mohallem, M. (2017). Horizontal Judicial Dialogue on Human Rights. En Müller, A. (ed.), Judicial Dialogue and Human Rights (67-113). Cambridge: Cambridge University Press. Disponible en: doi:10.1017/9781316780237.003.

Müller, A., y KJos, H. (2017). Introduction. En Müller, A. (ed.), Judicial Dialogue and Human Rights, Cambridge, Cambridge University Press. Disponible en: doi:10.1017/9781316780237.001.

NunEs, R. M. (2010). Ideational Origins of Progressive Judicial Activism: Colombian Constitutional Court and the Right to Health. Latin American Politics and Society, 52 (3). Disponible en: https://doi.org/10.1111/ j.1548-2456.2010.00090.x.

LAX, Jeffrey. (2011) The New Judicial Politics of Legal Doctrine. Annual Review of PoliticalScience 14, (2011). Disponible en: https://doi. org/10.1146/annurev.polisci.042108.134842. Google Scholar.

PÉREZ PERDOMO, Rogelio. (2012). Gente del derecho y cultura jurídica en América Latina, México, UNAM, Instituto de Investigaciones Jurídicas, 2012. Google Scholar.

Rodiles, A. (2016). The Law and Politics of the Pro Persona Principle in Latin America. En Aust, H. y NolTE, G. (eds.). The Interpretation of International Law by Domestic Courts. Uniformity, Diversity, Convergence. Oxford: Oxford University Press, 2016. Disponible en: DOI: 10.1093/acprof:oso/9780198738923.001.0001

SANDHOLTZ, W. (2015). How Domestic Courts Use International Law. Fordham International Law Journal, 38 (2).

SERNA DE LA GARZA, J. (2012). Impacto e implicaciones constitucionales de la globalización en el sistema jurídico mexicano. México: UNAM, Instituto de Investigaciones Jurídicas.

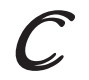

